\title{
Analysis of Certain Fruit Juices Using Microwave Techniques
}

\author{
Anil Lonappan ${ }^{*}$, Thomas Joachim Odhiambo Afullo², Williams Mark Uren Daniels ${ }^{3}$ \\ ${ }^{1}$ Department of Electrical Engineering, Mangosuthu University of Technology, Jacobs, Durban, South Africa \\ ${ }^{2}$ School of Electrical Electronic and Computer Engineering, Howard College, University of KwaZulu-Natal, Durban, South Africa \\ ${ }^{3}$ College of Health Sciences, School of Laboratory Medicine and Medical Sciences, University of KwaZulu-Natal, Durban, South Africa \\ Email: *lonappan@mut.ac.za
}

How to cite this paper: Lonappan, A., Afullo, T.J.O. and Daniels, W.M.U. (2017) Analysis of Certain Fruit Juices Using Microwave Techniques. Journal of Electromagnetic Analysis and Applications, 9, 123-134.

https://doi.org/10.4236/jemaa.2017.99011

Received: September 11, 2017

Accepted: September 24, 2017

Published: September 27, 2017

Copyright (C) 2017 by authors and Scientific Research Publishing Inc. This work is licensed under the Creative Commons Attribution International License (CC BY 4.0).

http://creativecommons.org/licenses/by/4.0/

\begin{abstract}
This paper discusses a novel method of analyzing the dielectric properties of juices at various frequencies of microwave band. The measurements were performed using a rectangular cavity with the perturbation technique at the S-band of microwave frequency with fresh juices made instantly from the fruits as well as collected from the packed fruit juices available in the market with the normal added preservatives in order to keep the increase of the shelf life. From the results, it is observed in the certain dielectric properties, the fresh juices samples and packed juices samples were varying and also varying over a period of time. This measurement method is simple and quick and can be used over a range of juices. These results prove a new method of determining the quality control of juices using microwave principles.
\end{abstract}

\section{Keywords}

Fruit, Juice, Cavity Perturbation, Shelf Life, Quality Control

\section{Introduction}

Fruits are normally sweet-tasting plant product, sometimes with seeds or without seeds, and most of them are edible in the raw state. Humans and animals make use of fruits as a source of food. In terms of the nutrition value, a fruit basically consists of macronutrients and micronutrients. The macronutrients like fiber and micronutrients like minerals, vitamins $\mathrm{C}$, thiamin, riboflavin, B-6, niacin, folate, A, and E. Phytochemicals like polyphenolics, carotenoids, and glucosinolates, will add nutritional value to a fruit [1] [2]. Mechanically squeezing or macerating a fruit will lead the preparation of a juice. Also though juice will have reduced macronutritional value, it can be made available at all times by 
addition of preservatives while fruits are available only at the designated period of the crop. So packed fruit juice have very high commercial value in today's world. But packed fruit juice will have added preservatives like ascorbic acid, carbon dioxide and in some cases colorants in order to increase its shelf life. The present study is an attempt to look into the dielectric properties of fresh juice as well as packed fruit juice at microwave frequencies.

At electromagnetic field, the dielectric property depends on water content, frequency, intensity of the field as well as temperature. As the temperature increases, more and more dipoles are free to oscillate, thereby there will be an increase in dielectric characteristics. The dielectric property is represented by dielectric constant and dielectric loss factor. The dielectric constant decides how much incident energy can go through the material and dielectric loss determines how much energy can be absorbed within the material. In reality, the energy absorption as well as heat dissipation in a material is purely on the basis of specific characteristics like density, water content, composition and surface structure as well as bulk characteristics like shape, size and porosity [3].

The microwave technology is used in various applications in food industry because of the energy is directly transferred to the materials which have moisture content rather than the conventional conduction mechanism which is a slow process. Over the conventional processing methods, microwave technology provides a rapid, clean and efficient heating over a wide range of temperatures. Electromagnetic energy is directly transferred to and absorbed by the moisture content material being processed in microwave processing. Thus, microwave energy can penetrate, thereby producing heat makes microwave technology better advantages when compared with other conventional heat processing techniques so that it is used in reheating, pasteurization, curing, freeze-drying, thawing, sterilization, tempering, baking, pre-cooking, dehydration and blanching [4] [5] [6] [7] [8].

Various measurement methods are used to measure the complex permittivity of a material and the method of choice depends on factors like the nature of the samples under study and the frequency range used [9] [10] [11] [12]. When only very small volumes of the sample are available, the cavity perturbation technique is an attractive option as it requires only minute volumes for the measurement [13]. This makes it suitable for the dielectric study of juices needed. However, no data is currently available for the complex permittivity of fresh juices and packed juices in the literature. In this study, the rectangular cavity perturbation technique has been employed for the measurement of the dielectric properties of freshly prepared juices as well as packed juices available in the market with the chemically added preservatives in order to increase its shelf life. The frequency of measurement is made between 2 to $4 \mathrm{GHz}$. It is observed that a more appreciable change in the dielectric properties of packed fruit juice than the fresh samples at the extended period of time. This microwave measurement technique is very simple, quick and the results are promising as this new method can be 
used in the quality control of packed fruit juices.

\section{Sample Preparation}

The study was conducted on pineapple, orange, naartjie, grape, apple, lemon, guava, lychee, strawberry and mango. Fresh fruits are obtained from the market and made into juice by mechanically squeezing just before the measurements. Similarly the packed fruit juice from the market which are kept at temperature at $4^{\circ} \mathrm{C}$ to $8^{\circ} \mathrm{C}$ were used for the measurements. The juices were filled into the capillary bulb for microwave measurements and the measurements were made at $20^{\circ} \mathrm{C}$.

\section{Material and Methods}

The experimental set-up consists of a transmission type S-band rectangular cavity resonator, Rohde \& Schwarz ZVL network analyzer. The cavity resonator is a transmission line with one or both ends closed. Figure 1 shows the experimental setup. The numbers of resonant frequencies are determined by the length of the resonator. The resonator in this set-up is excited in the $\mathrm{TE}_{10 \mathrm{p}}$ mode. The sample holder which is made of glass in the form of a capillary tube flared to a disk shaped bulb at the bottom is placed into the cavity through the non-radiating cavity slot, at broader side of the cavity which can facilitate the easy movement of the holder. The resonant frequency $f_{o}$ and the corresponding quality factor $Q_{o}$ of the cavity at each resonant peak with the empty sample holder placed at the maximum electric field are noted. The same holder filled with known amount of sample under study is again introduced into the cavity resonator through the non-radiating slot. The resonant frequencies of the sample loaded cavity are selected and the position of the sample is adjusted for maximum perturbation (i.e. maximum shift of resonant frequency with minimum amplitude for the peak). The new resonant frequency fs and the quality factor $Q_{s}$ are noted. The same procedure is repeated for other resonant frequencies. The measurement is made from $2 \mathrm{GHz}$ to $4 \mathrm{GHz}$ of S-band in microwave region [14].

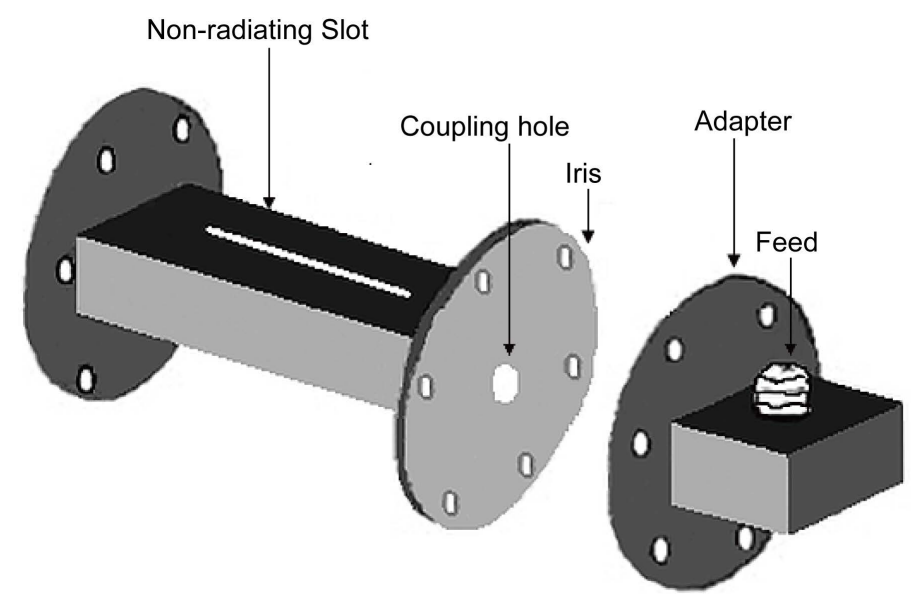

Figure 1. Experimental set up. 


\section{Theory}

Theoretical analysis for the determination of complex permittivity of materials using rectangular waveguide cavity: When a small sample is inserted in a cavity which has the electric field $E_{0}$ and magnetic field $H_{0}$ in the unperturbed state, the fields in the interior of the object are $E$ and $H$. Beginning with Maxwell's equations, Bethe and Schwinger [15] obtained an expression for the resonant frequency shift. For lossless sample, the variation of resonant frequency is given by Harrington [16] as

$$
\frac{\omega-\omega_{0}}{\omega}=-\frac{\int\left(\Delta \varepsilon E \cdot E_{0}^{*}+\Delta \mu H \cdot H_{0}^{*}\right) \mathrm{d} \tau}{\int\left(\varepsilon E \cdot E_{0}^{*}+\mu H \cdot H_{0}^{*}\right) \mathrm{d} \tau}
$$

where $\varepsilon$ and $\mu$ are the permittivity and permeability of the medium in the unperturbed cavity respectively and $\mathrm{d} \tau$ is the elemental volume. $\Delta \varepsilon$ and $\Delta \mu$ are changes in the above quantities due to introduction of the sample in the cavity. Without affecting the generality of Maxwell's equations, the complex frequency shift due to a lossy sample in the cavity is given by Waldron [17]

$$
-\frac{\delta \Omega}{\Omega} \approx \frac{\left(\bar{\varepsilon}_{r}-1\right) \varepsilon_{0} \int_{V_{s}} E \cdot E_{0}^{*} \mathrm{~d} V+\left(\bar{\mu}_{r}-1\right) \mu_{0} \int_{V_{s}} H \cdot H_{0}^{*} \mathrm{~d} V}{\int_{V_{c}}\left(D_{0} \cdot E_{0}^{*}+B_{0} \cdot H_{0}^{*}\right) \mathrm{d} V}
$$

Two approximations are made in applying Equation (2), based on the assumption that fields in the empty part of the cavity are negligibly changed by the insertion of the sample and that the fields in the sample are uniform over its volume. Both these assumptions can be considered valid if the object is sufficiently small relative to the resonant wavelength. The negative sign in Equation (2) indicates that by introducing the sample the resonant frequency is lowered. Because the permittivity of practical materials is complex, the resonant frequency should also be considered as complex. In Equation (2), the $\mathrm{d} \Omega$ is the complex frequency shift. $B_{0}, H_{0}, D_{0}$ and $E_{0}$ are the fields in the unperturbed cavity. $E$ and $H$ are the fields in the interior of the sample. $\bar{\varepsilon}_{r}=\vec{\varepsilon}_{r}-j \vec{\varepsilon}_{r}^{\prime \prime}$ and $\bar{\mu}_{r}=\bar{\mu}_{r}^{\prime}-j \bar{\mu}_{r}^{\prime \prime}$ and $V_{c}$ and $V_{s}$ are the volumes of the cavity and sample respectively. In terms of energy, the numerator of Equation (2) represents the energy stored in the sample and the denominator represents the total energy stored in the cavity. The total energy $W=W_{e}+W_{m}=2 W_{e}=2 W_{m}$. When a dielectric sample is introduced at the position of maximum electric field only the first term in the numerator is significant, since a small change in $\varepsilon$ at a point of zero electric field or a small change in $\mu$ at a point of zero magnetic field does not change the resonance frequency. Thus Equation (2) can be reduced to

$$
-\frac{\delta \Omega}{\Omega} \approx \frac{\left(\bar{\varepsilon}_{r}-1\right) \int_{V_{s}} E \cdot E_{0 \max }^{*} \mathrm{~d} V}{2 \int_{V_{c}}\left|E_{0}\right|^{2} \mathrm{~d} V}
$$

Let $Q_{0}$ be the quality factor of the cavity in the unperturbed condition and 
$Q_{s}$ the Q-factor of the cavity loaded with the object. The complex frequency shift is related to measurable quantities by [18]

$$
\frac{\delta \Omega}{\Omega} \approx \frac{\delta \omega}{\omega}+\frac{j}{2}\left[\frac{1}{Q_{s}}-\frac{1}{Q_{0}}\right]
$$

Equating the real and imaginary terms of Equations (3) and (4) we get

$$
\begin{aligned}
& -\frac{f_{s}-f_{0}}{f_{s}}=\frac{\left(\varepsilon_{r}^{\prime}-1\right) \int_{V_{s}} E \cdot E_{0 \max }^{*} \mathrm{~d} V}{2 \int_{V_{c}}\left|E_{0}\right|^{2} \mathrm{~d} V} \\
& \frac{1}{2}\left[\frac{1}{Q_{s}}-\frac{1}{Q_{0}}\right]=\frac{\varepsilon_{r}^{\prime \prime} \int_{V_{s}} E \cdot E_{0 \text { max }}^{*} \mathrm{~d} V}{2 \int_{V_{c}}\left|E_{0}\right|^{2} \mathrm{~d} V}
\end{aligned}
$$

We may assume that $E \approx E_{0}$ and the value of $E_{0}$ in $T E_{10 p}$ mode as $E_{0}=E_{0 \max } \sin \left(\frac{m \prod x}{a}\right) \sin \left(\frac{p \prod z}{d}\right)$ where $a$ is the broader dimension of the waveguide and $d$ is the length of the cavity. Integrating and rearranging the above equations we get

$$
\begin{aligned}
& \varepsilon_{r}^{\prime}-1=\frac{f_{0}-f_{s}}{2 f_{s}}\left(\frac{V_{c}}{V_{s}}\right) \\
& \varepsilon_{r}^{\prime \prime}=\frac{V_{c}}{4 V_{s}}\left[\frac{1}{Q_{s}}-\frac{1}{Q_{0}}\right]
\end{aligned}
$$

If the frequency shift is measured from the resonance frequency $f_{t}$ of the cavity loaded with empty capillary rather than that with empty cavity alone the above equations become

$$
\begin{gathered}
\varepsilon_{r}^{\prime}-1=\frac{f_{t}-f_{s}}{2 f_{s}}\left(\frac{V_{c}}{V_{s}}\right) \\
\varepsilon_{r}^{\prime \prime}=\frac{V_{c}}{4 V_{s}}\left[\frac{1}{Q_{s}}-\frac{1}{Q_{t}}\right]
\end{gathered}
$$

$Q_{t}$ is the quality factor of the cavity loaded with empty capillary. $f_{s}$ and $Q_{s}$ are the resonance frequency and quality factor of the cavity loaded with capillary containing the sample material.

Theory for the determination of conductivity of the materials:

For a dielectric material having non-zero conductivity, the Ampere's law in phasor form as

$$
\nabla \times H=(\sigma+j \omega \bar{\varepsilon}) E=\left(\sigma+j \omega \varepsilon^{\prime \prime}\right) E+j \omega \varepsilon^{\prime} E
$$

where $\varepsilon=\varepsilon^{\prime}-j \varepsilon^{\prime \prime}$ is the absolute permittivity of the medium.

The loss tangent 


$$
\tan \delta=\frac{\sigma+\omega \varepsilon^{\prime \prime}}{\omega \varepsilon^{\prime}}
$$

For dielectrics $\sigma=0$ then Equation (12a) becomes

$$
\tan \delta=\frac{\varepsilon^{\prime \prime}}{\varepsilon^{\prime}}
$$

$\sigma_{e}=\sigma+\omega \varepsilon^{\prime \prime}$, is the effective conductivity of the medium.

But

$$
\tan \delta=\frac{1}{Q_{m}}=\left[\frac{1}{Q_{s}}-\frac{1}{Q_{t}}\right]
$$

where is $Q_{m}$ is the loaded $Q$-factor of the cavity with sample alone.

The effective conductivity

$$
\sigma_{e}=\frac{\omega \varepsilon^{\prime}}{Q_{m}}=\frac{\omega \varepsilon_{r}^{\prime} \varepsilon_{0}}{Q_{m}}
$$

When $\sigma$ is very small, the effective conductivity is reduced to

$$
\sigma_{e}=\omega \varepsilon^{\prime \prime}=2 \pi f \varepsilon_{0} \varepsilon_{r}^{\prime \prime}
$$

\section{Results and Discussion}

The microwave experiment in fruit juices were done using cavity perturbation technique collected from freshly prepared juices as well as packed juices and the results were shown in Figures 2-13. From Figures 2-4 it is observed that freshly prepared juices as well as packed juices exhibit almost similar ranges of dielectric constant even though the measurements were made at different intervals of time. From Figures 5-7 shows the dielectric loss or conductivity of freshly prepared juices. These results are shows that the conductivities are consistent over the extended period of time. From Figures 8-10 shows the dielectric constant of packed

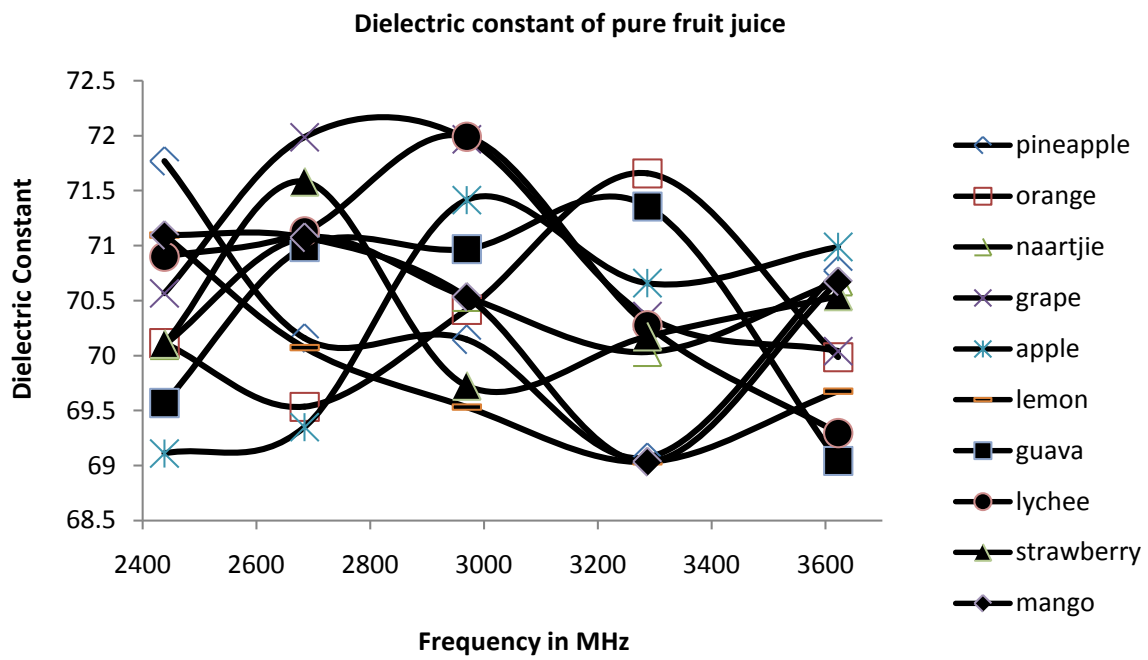

Figure 2. Variation of dielectric constant of pure fruit juice. 


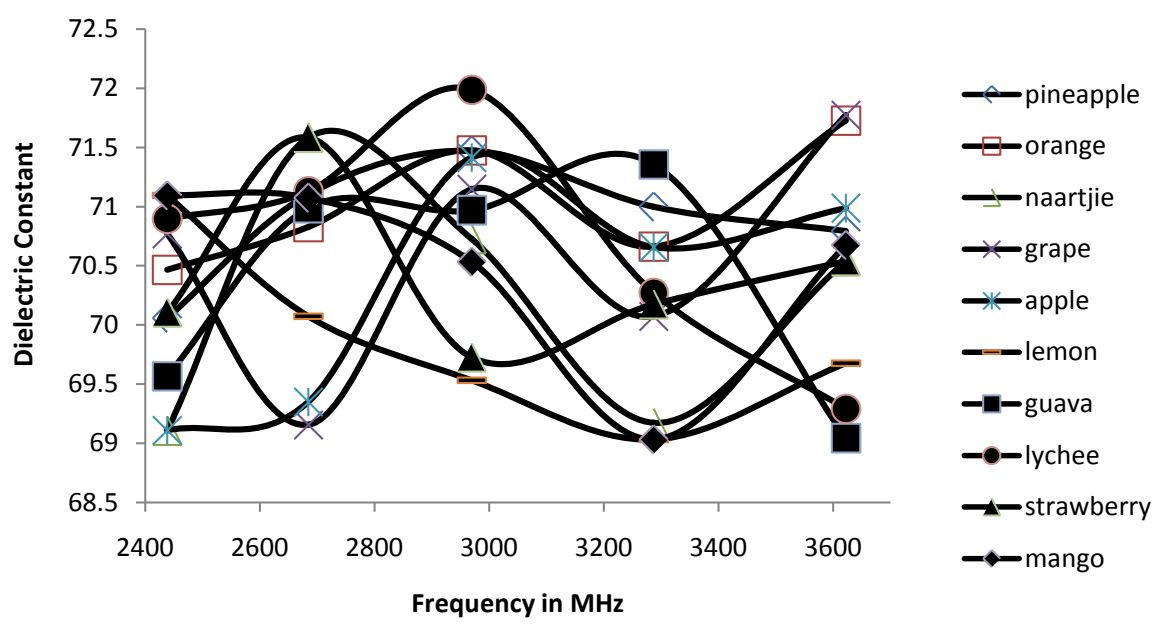

Figure 3. Variation of dielectric constant of pure fruit juice after 1 hour.

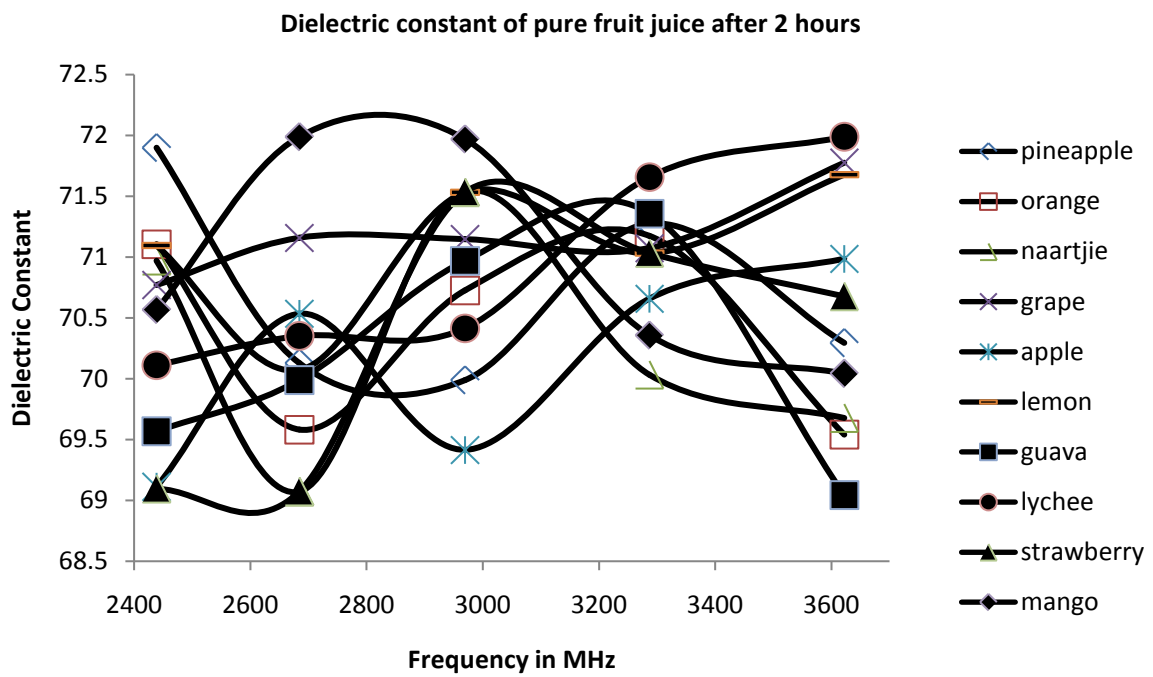

Figure 4. Variation of dielectric constant of pure fruit juice after 2 hours.

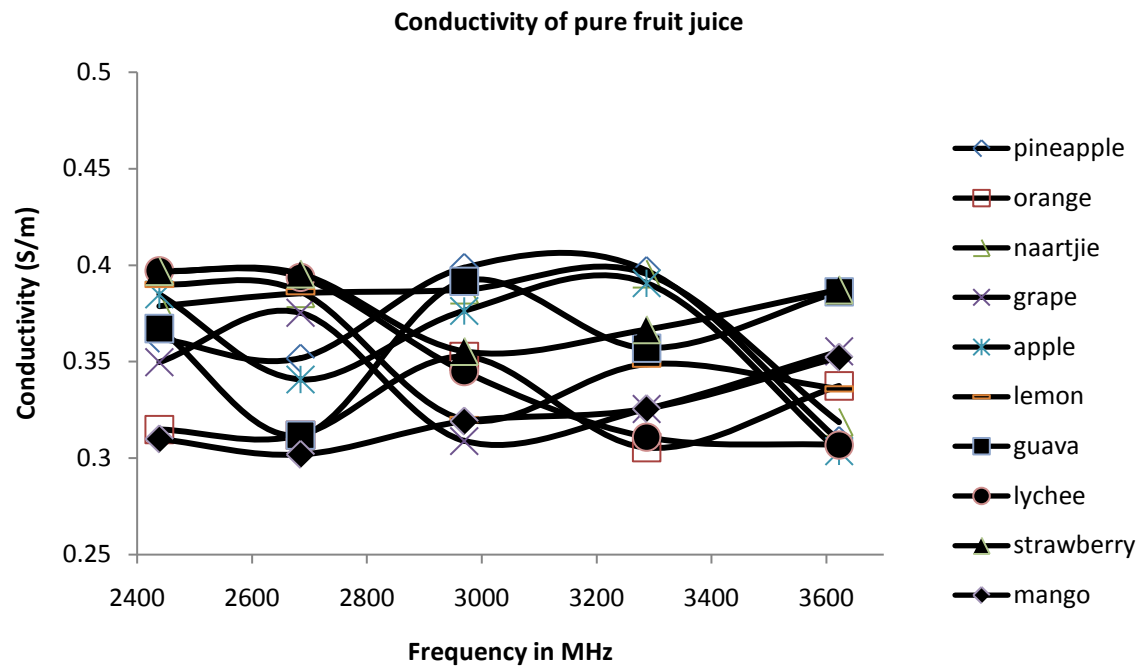

Figure 5. Variation of conductivity of pure fruit juice. 


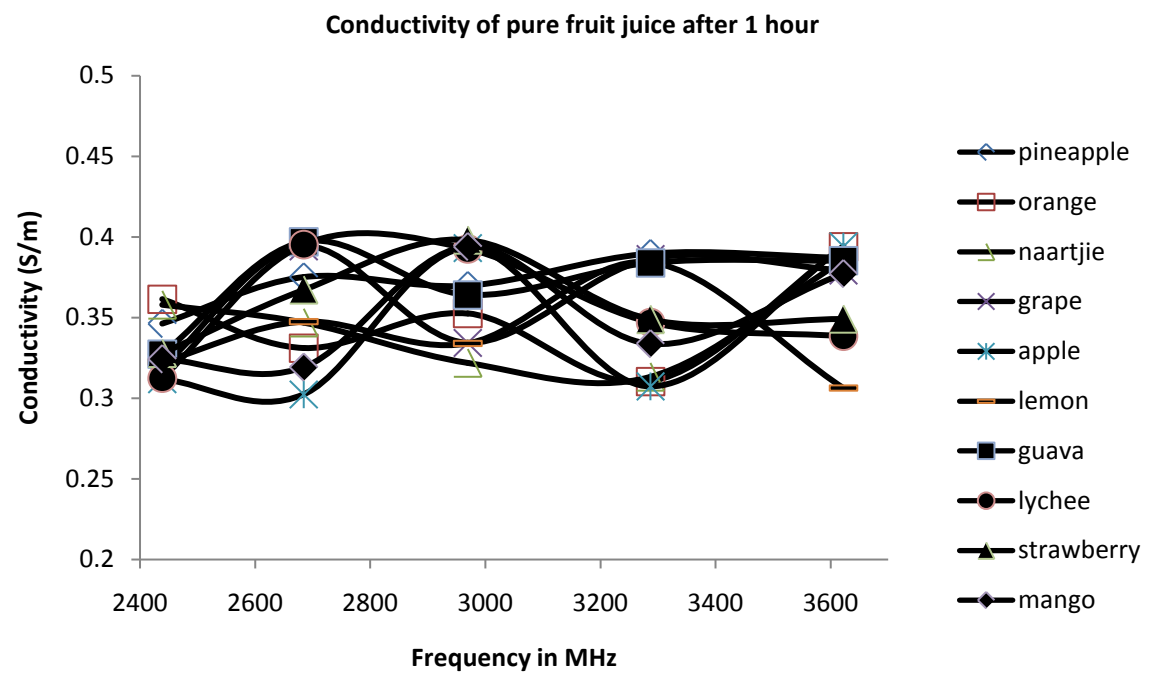

Figure 6. Variation of conductivity of pure fruit juice after 1 hour

Conductivity of pure fruit juice after $\mathbf{2}$ hours

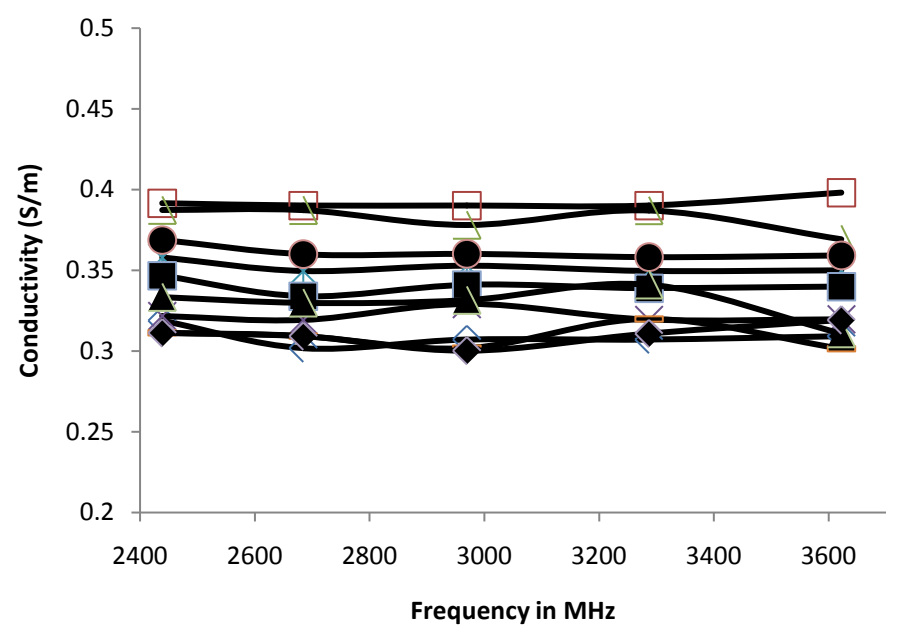

—-pineapple

-

$\longrightarrow$ naartjie

$\rightarrow$ grape

$\rightarrow$-apple

--lemon

- - -guava

-๑-lychee

$\rightarrow$-strawberry

$-\infty$ mango

Figure 7. Variation of conductivity of pure fruit juice after 2 hours.

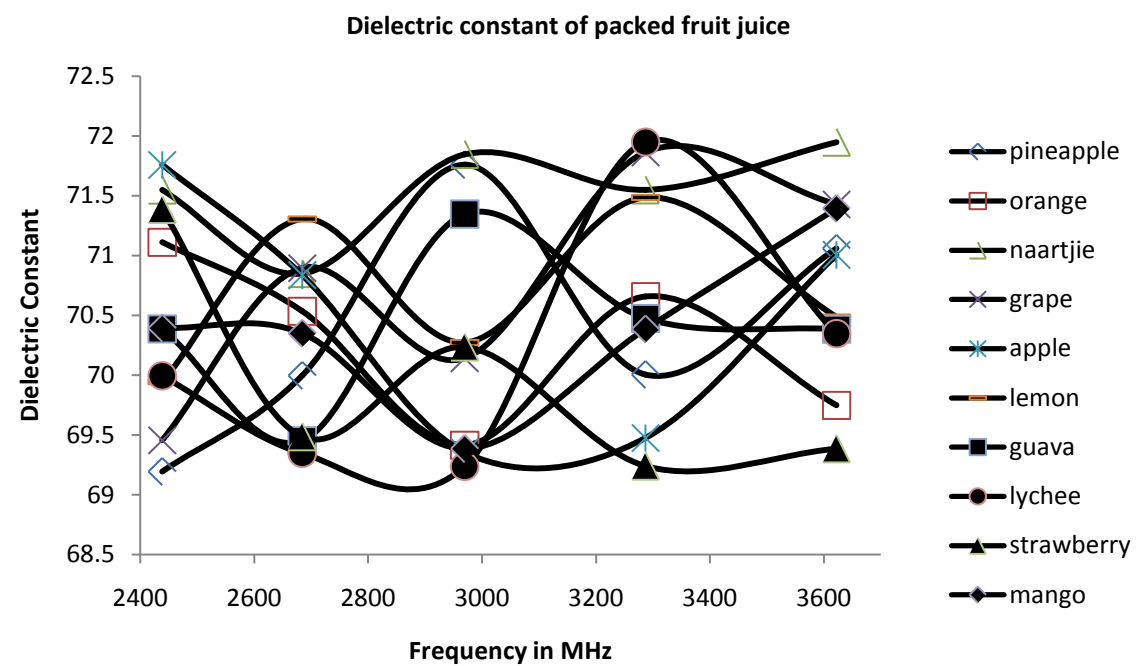

Figure 8. Variation of dielectric constant of packed fruit juice. 


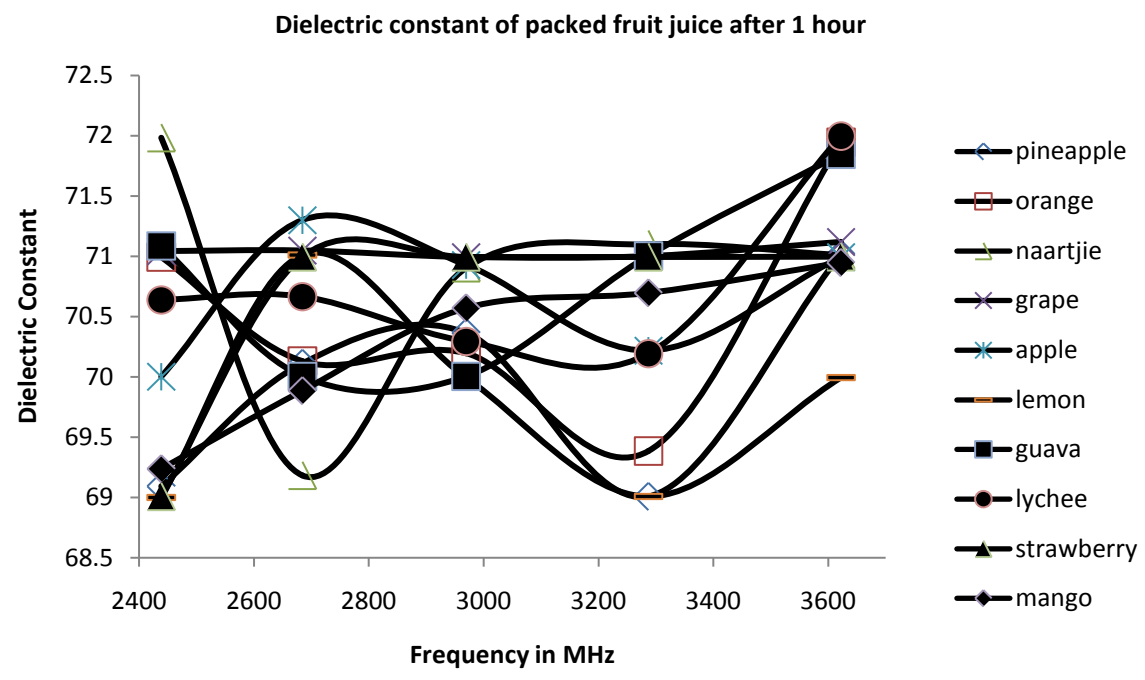

Figure 9. Variation of dielectric constant of packed fruit juice after 1 hour.

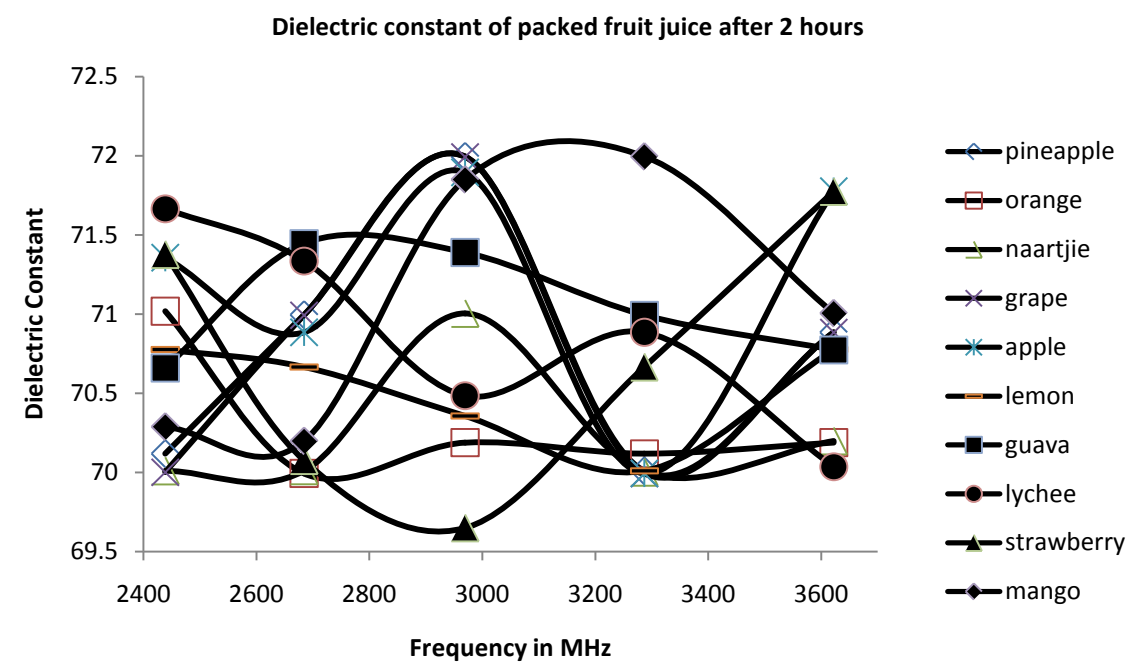

Figure 10. Variation of dielectric constant of packed fruit juice after 2 hours.

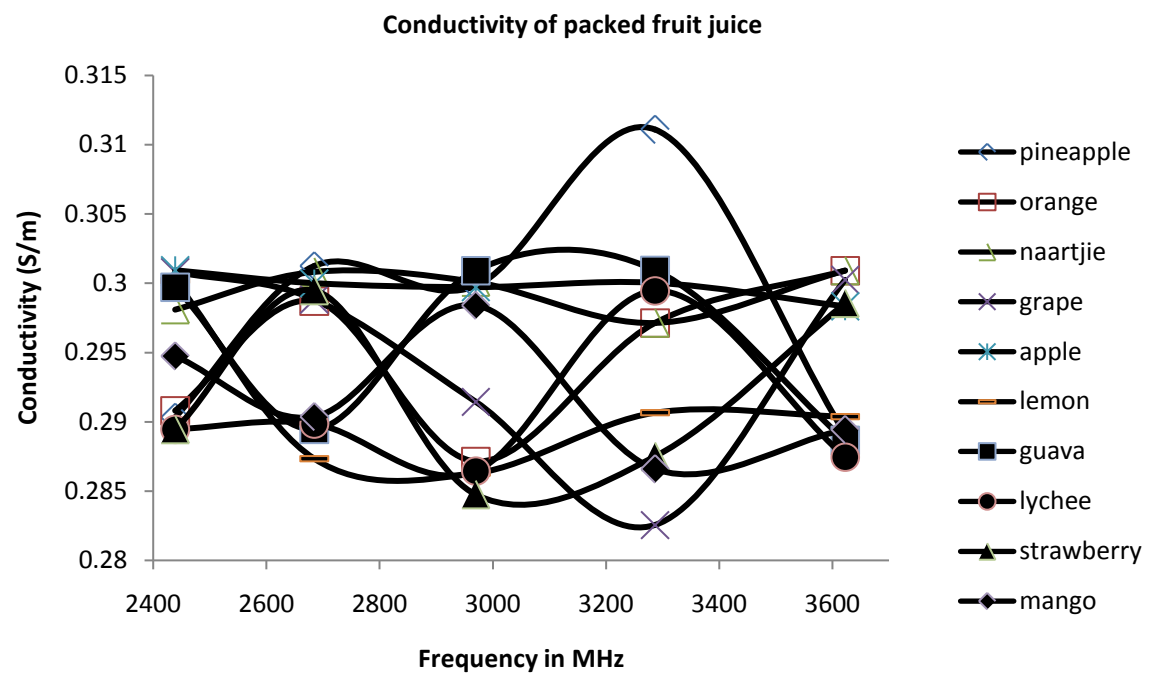

Figure 11. Variation of conductivity of packed fruit juice. 


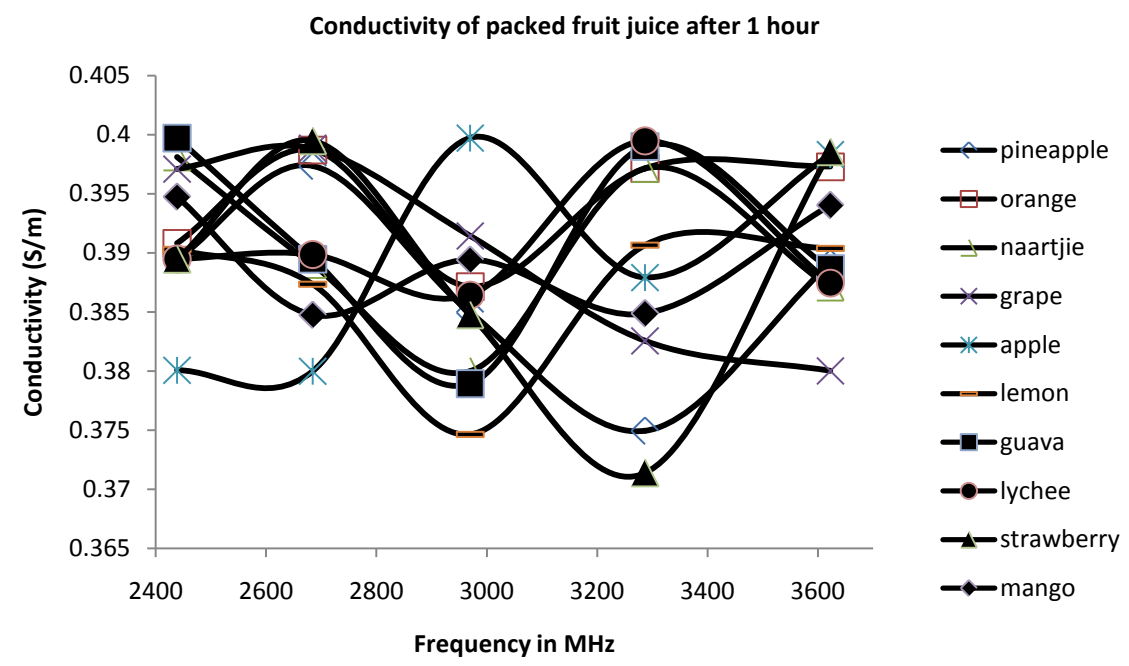

Figure 12. Variation of conductivity of packed fruit juice after 1 hour.

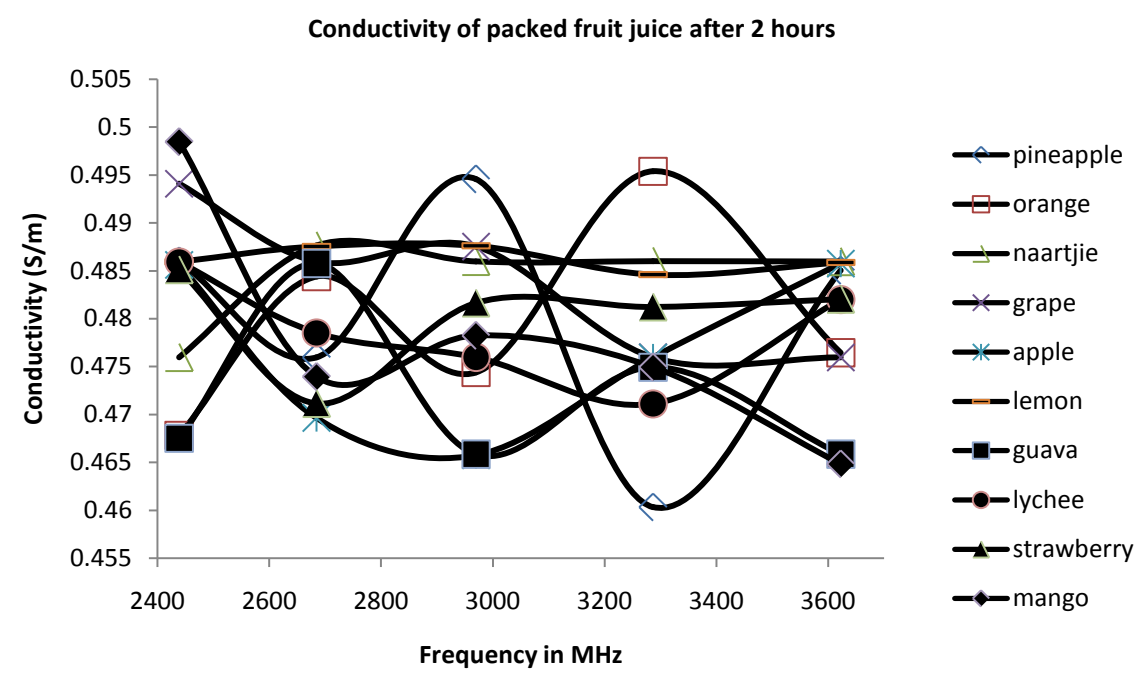

Figure 13. Variation of conductivity of packed fruit juice after 2 hours.

fruit juice at different time intervals. These results suggest that the dielectric constant is similar for the different intervals of time. From Figures 11-13 shows the conductivities of packed fruit juice over the extended period of time. These results show that there is a distinct variation of conductivities over the period of time as well as with that of freshly prepared juices. Packed juices exhibit higher conductivity than the freshly prepared juices because of the presence of added preservatives which is necessary to increase its shelf life. So it is very critical that packed fruit juice should be consumed immediately after it is opened. Thus in the $\mathrm{S}$ band of microwave (ISM band), freshly prepared fruit juices and packed fruit juice were studied and exhibit distinct variation of conductivity even after certain intervals of time.

\section{Conclusion}

The microwave characterization has been performed in the freshly prepared 
juices as well as packed fruit juices using the cavity perturbation technique. The cavity perturbation technique is quick, simple, and accurate and it requires very low volume of sample for measuring the dielectric properties of samples like juices. The limitation of this method is that measurement can lead to more accurate results on liquid samples than on solid samples. From the results, it is observed in the certain dielectric properties, the fresh juices samples and packed juices samples were varying and also varying over a period of time. This measurement method is simple and quick and can be used over a range of juices. These results prove a new method of determining the quality control of juices using microwave principles.

\section{References}

[1] Franke, A.A., Cooney, R.V., Henning, S.M. and Custer, L.J. (2005) Bioavailability and Antioxidant Effects of Orange Juice Components in Humans. Journal of Agricultural Food Chemistry, 53, 5170-5178. https://doi.org/10.1021/jf050054y

[2] Kurowska, E.M., Spence, J.D., Jordan, J., Wetmore, S., Freeman, D.J., Piché, L.A. and Serratore, P. (2000) HDL-Cholesterol-Raising Effect of Orange Juice in Subjects with Hypercholesterolemia. American Journal Clinical Nutrition, 72, 1095-1100.

[3] Ahmed, J. and Ramaswamy, H.S. (2007) Handbook of Food Preservation. 2nd Edition, Taylor \& Francis Group, CRC Press, 691-711.

[4] Fratianni, A., Cinquanta, L. and Panfili, G. (2010) Degradation of Carotenoids in Orange Juice during Microwave Heating. Food Science and Technology, 43, 867-871. https://doi.org/10.1016/j.lwt.2010.01.011

[5] Cinquanta, L., Albanese, D., Cuccurullo, G. and Dimatteo, M. (2010) Effect on Orange Juice of Batch Pasteurization in an Improved Pilot-Scale Microwave Oven. Journal of Food Science, 75, 46-50. https://doi.org/10.1111/j.1750-3841.2009.01412.x

[6] Prakash, A., Kim, H.J. and Taub, I.A. (1997) Assessment of Microwave Sterilization of Foods Using Intrinsic Chemical Markers. Journal of Microwave Power Electromagnetic Energy, 32, 50-57. https://doi.org/10.1080/08327823.1997.11688323

[7] Ohlsson, T. and Bengtsson, N.E. (1975) Dielectric Food Data for Microwave Sterilization Processing. Journal of Microwave Power Electromagnetic Energy, 10, 93-108.

[8] Tajchakavit, S. and Ramaswamy, H.S. (1997) Thermal vs. Microwave Inactivation Kinetics of Pectin Methylesterase in Orange Juice under Batch Mode Heating Conditions. Food Science and Technology, 30, 85-93.

[9] Ghodgaonkar, D.K., Varadan, V.V. and Varadan, V.K. (1990) Free Space Measurement of Complex Permittivity and Complex Permeability of Magnetic Materials at Microwave Frequencies. IEEE Transactions on Instrumentation and Measurement, 39, 387-394. https://doi.org/10.1109/19.52520

[10] Ghodgaonkar, D.K., Varadan, V.V. and Varadan, V.K. (1989) A Free Space Method for Measurement of Dielectric Constant and Loss Tangents at Microwave Frequencies. IEEE Transaction on Instrumentation and Measurement, 38, 789-793. https://doi.org/10.1109/19.32194

[11] Barry, W. (1986) A Broadband, Automated, Stripline Technique for the Simultaneous Measurement of Complex Permittivity and Complex Permeability. IEEE Transaction on Microwave Theory and Techniques, 34, 80-84.

https://doi.org/10.1109/TMTT.1986.1133283 
[12] Abbas, Z., Pollard, R.D. and Kelsall, R.W. (1998) A Rectangular Dielectric Waveguide Technique for Determination of Permittivity of Materials at W-Band. IEEE Transactions on Microwave Theory and Techniques, 46, 2011-2015. https://doi.org/10.1109/22.739275

[13] Mathew, K.T. (2005) Perturbation Theory. In: Encyclopedia of RF and Microwave Engineering, Wiley-Interscience, Vol. 4, 3725-3735. https://doi.org/10.1002/0471654507.eme309

[14] Lonappan, A. (2012) Novel Method of Detecting H1N1 using Microwaves. Journal of Biomedical Science and Engineering, 5.

[15] Bethe, H.A. and Schwinger, J. (1943) NDRC Report D1-117. Cornell University.

[16] Harrington, R.F. (1961) Time-Harmonic Electromagnetic Fields. McGraw-Hill, New York, 317.

[17] Waldron, R.A. (1960) Perturbation Theory of Resonant Cavities. Proceedings of Institution of Electrical Engineering, 107C, 272-274. https://doi.org/10.1049/pi-c.1960.0041

[18] Waldron, R.A. (1969) Theory of Waveguides and Cavities. Maclaran and Sons, London, 75.

Submit or recommend next manuscript to SCIRP and we will provide best service for you:

Accepting pre-submission inquiries through Email, Facebook, LinkedIn, Twitter, etc. A wide selection of journals (inclusive of 9 subjects, more than 200 journals)

Providing 24-hour high-quality service

User-friendly online submission system

Fair and swift peer-review system

Efficient typesetting and proofreading procedure

Display of the result of downloads and visits, as well as the number of cited articles

Maximum dissemination of your research work

Submit your manuscript at: http://papersubmission.scirp.org/

Or contact jemaa@scirp.org 